\title{
Mitarbeiter des Heftes
}

Prof. Dr. Manfred Eibelshäuser, Präsident des Hessischen Rechnungshofs, Eschollbrücker Straße 27, D-64295 Darmstadt

Guido Eitberger, Diakonisches Werk der EKD e.V., Postfach 330220, D-14172 Berlin

Prof. Dr. Werner W. Engelhardt, Hochwaldstraße 38, D-50935 Köln

Mathias Häfner, MVV Energie AG, Luisenring 59, D-68159 Mannheim

Dr. Peter Mühlhausen, Bodelschwinghstraße 2, D-34119 Kassel

RA Horst Müller, Theresienstraße 13, D-80333 München

Dr. Karsten Nowak, Eschollbrücker Straße 27, D-64295 Darmstadt

Prof. Dr. Dr. h.c. Günter Püttner, Universität Tübingen, Lehrstuhl für Staats- und Verwaltungsrecht, Öffentliches Wirtschaftsrecht, Wilhelmstraße 7, D-72074 Tübingen

Dr. Markus Rückert, Vorsitzender des Vorstands des Collegium Augustinum, Stiftsbogen 74, D-81375 München

Thomas Sachsenweger, MVV Energie AG, Luisenring 59, D-68159 Mannheim

Prof. Dr. Dirk Schiereck, European Business School, Stiftungslehrstuhl Bank- und Finanzmanagement, Schloss Reichartshausen, D-65375 Oestrich-Winkel

Georg von Schnurbein, Universität Freiburg, Verbandsmanagement Institut, Postfach 1559, $\mathrm{CH}-1701$ Freiburg

Dr. Stephanie Scholz, Diakonisches Werk der EKD e.V., Postfach 330220, D-14172 Berlin

Prof. Dr. Frank Schulz-Nieswandt, Universität zu Köln, Seminar für Sozialpolitik, AlbertusMagnus-Platz, D-50931 Köln

Prof. Dr. Peter Schwarz, Universität Freiburg, Verbandsmanagement Institut, Postfach 1559, $\mathrm{CH}-1701$ Freiburg

Prof. Dr. Hans-Peter Schwintowski, Humboldt-Universität zu Berlin, Lehrstuhl für Bürgerliches Recht, Handels- Wirtschafts- und Europarecht, Unter den Linden 6, D-10099 Berlin

Prof. Dr. Bernd Stauss, Katholische Universität Eichstätt-Ingolstadt, Lehrstuhl für Allgemeine Betriebswirtschaftslehre, Absatzwirtschaft und Marketing, Ostenstraße 26, D-85072 Eichstätt

Prof. Dr. Franz Wirl, Betriebswirtschaftszentrum der Universität Wien, Lehrstuhl für Industrie, Energie und Umwelt, Brünner Straße 72, A-1210 Wien

Redaktion

Anke Reile, Universität Mannheim, L 5,4 am Schloss, D-68131 Mannheim, Tel. 0621/181-1725, e-mail: zoegu@bwl.uni-mannheim.de 